\title{
THE FINITE CONVOLUTION TRANSFORM
}

\author{
BY \\ JEROME BLACKMAN(1) AND HARRY POLLARD( $\left.{ }^{2}\right)$
}

1. Introduction. There exists a large literature concerning convolution transforms on the whole real line, but the corresponding problem for semiinfinite intervals has received little attention. The problem is that of determining a function $\phi$ from the relation

$$
f(x)=\int_{0}^{x} \phi(x-t) K(t) d t, \quad 0 \leqq x<\infty
$$

given $f$ and the kernel $K$.

If $\phi$ and $K$ have suitable behavior at infinity the classical solution by Laplace transforms is available. This method of solution, however, determines $\phi$ from a knowledge of $f(x)$ for all $x$ in $0 \leqq x<\infty$, whereas the form of (1) suggests that the determination of $\phi(x)$ in an interval $0 \leqq x<a$ should use the values of $f(x)$ only in this interval. This suggestion is confirmed by a theorem of Titchmarsh [1, p. 327] concerning the equation

$$
0=\int_{0}^{x} \phi(x-t) K(t) d t, \quad 0 \leqq x<a,
$$

where $a$ is a fixed positive number. It is a consequence of his theorem that if $\phi$ and $K$ are locally integrable and $K(x) \neq 0$ in a neighborhood $0 \leqq x<\eta$ of the origin, then the only solution of (2) is $\phi \equiv 0$.

The main object of this paper is to give a solution of the equation in (1) for an interval $0 \leqq x<a$. Clearly this solves the problem for the whole interval $0 \leqq x<\infty$. There is no added difficulty in solving the more general equation

$$
f(x)=\int_{0}^{x} \phi(x-t) d k(t), \quad 0 \leqq x<a,
$$

and this is what we shall do. It is supposed that $k(t)$ is of bounded variation in each interval $0 \leqq x \leqq a_{1}, 0<a_{1}<a$, and that $\phi$ is Borel measurable in $0 \leqq x<a$.

2. Assumptions. In solving (3) the following assumptions will be made about $k$ and $\phi$.

Presented to the Society, April 26, 1958; received by the editors August 19, 1957.

(1) Research under contract with the Office of Naval Research.

(2) This research was supported by the United States Air Force under Contract No. AF18(600)-685 monitored by the Office of Scientific Rescarch. 
A1. $k$ has positive variation near 0 ;

2. $k(0)=0$;

3. $k$ is real-valued.

B1. $\phi \in C^{2}(0 \leqq x<a)$;

2. $\phi(0)=\phi^{\prime}(0)=0$.

Assumption A1 is necessary since otherwise (3) does not determine $\phi$. A2 can be achieved by replacing $k(t)$ with $k(t)-k(0)$ in (3). If $k$ is not realvalued to begin with, (3) can be replaced by a new equation of the same form for which the kernel is real-valued. The procedure is this: Define

$$
k_{1}(x)=\int_{0}^{x} k(x-t) d \bar{k}(t)
$$

and

$$
f_{1}(x)=\int_{0}^{x} f(x-t) d \bar{k}(t)
$$

where $\bar{k}$ is the complex-conjugate of $k$. Then

$$
f_{1}(x)=\int_{0}^{x} \phi(x-t) d k_{1}(t), \quad 0 \leqq x<a,
$$

and $k_{1}$ is real because of $\mathrm{A} 2$.

Assumptions B1 and B2 are satisfied by taking the precautionary measure of integrating (3) from 0 to $x$ three times before starting.

3. A study of the kernel $k$. Because of A1 we can choose a number $b$, $0<b<a / 2$ so that

$$
B=\int_{0}^{2 b} d k(u) \neq 0 .
$$

An important step in solving (3) is the determination of $\phi(x)$ in $0 \leqq x<b$, and thus depends on a preliminary study of the Laplace transform

$$
\hat{k}(s) \equiv \hat{k}_{b}(s)=\int_{0}^{2 b} e^{-s u} d k(u) .
$$

$\hat{k}$ is an entire function. Because of (4) it has the form

$$
\hat{k}(s)=B e^{-c^{\prime} s} \prod_{z}\left(1-\frac{s}{z}\right) e^{s / z},
$$

where $c^{\prime}$ is a constant and the product is taken over the roots $z$ of $\hat{k}$. Since $k$ is real the roots of $\hat{k}$ appear in conjugate-complex pairs unless they are real. Let $\alpha$ denote the real zeros and $\beta$ the complex zeros with positive imaginary part. 
$\hat{k}(s)$ is bounded in the right half-plane and $e^{2 b s} \hat{k}(s)$ in the left. Consequently the two series

$$
\sum_{\alpha} \frac{1}{|\alpha|} \text { and } \sum_{\beta} \frac{\cos (\arg \beta)}{|\beta|}
$$

converge, [2, p. 131]. (6) may therefore be written

$$
\hat{k}(s)=B e^{-c s} \prod_{\alpha}\left(1-\frac{s}{\alpha}\right) \prod_{\beta}\left(1-\frac{s}{\beta}\right)\left(1-\frac{s}{\bar{\beta}}\right) .
$$

An argument of Titchmarsh [1, p. 323], shows that because of A1 and (5) $c$ is real and satisfies

$$
0 \leqq c \leqq b .
$$

It will be necessary to arrange the product (8) in another order, the justification coming from the convergence of the two series (7). Define this function of a complex variable $w$ :

$$
S(w)= \begin{cases}\left(2\left[(I w)^{2}-(R w)^{2}\right]\right)^{1 / 2}, & |I w| \geqq|R w|, \\ -1, & |I(w)|<|R w| .\end{cases}
$$

The roots of $\hat{k}$ are decomposed into classes $A_{n}, n \equiv 0,1,2, \cdots$ according to the rule

$$
z \in A_{n} \text { if } n-1 \leqq S(z)<n .
$$

The following lemma is trivial.

Lemma 1. If $t$ is real then $|1-i t / z| \geqq 1$ for real zeros $z$. Also

$$
\left|\left(1-\frac{i t}{z}\right)\left(1-\frac{i t}{\bar{z}}\right)\right| \geqq 1
$$

if and only if $|t| \geqq S(z)$.

(8) may now be rearranged as

$$
k(s)=B e^{-c s} \prod_{j=0}^{\infty} \prod_{z \in A_{j}}\left(1-\frac{s}{z}\right)\left(1-\frac{s}{\bar{z}}\right) .
$$

An empty product is interpreted as unity and the prime' in the inner product means that only a single factor is written when $z$ is real. (This can occur only for $j=0$.) For a fixed $j$ the order of interior factors does not matter and we regard them as fixed once and for all, say by absolute value. For a fixed $j$ the zeros $z$ belonging to $A$; will be denoted generally by $z_{1}, z_{2}, \cdots$, thus avoiding the use of a double subscript. The last formula is conveniently broken up into 


$$
\begin{aligned}
g_{j}(s) & =\prod_{z \in A_{j}}\left(1-\frac{s}{z}\right)\left(1-\frac{s}{\bar{z}}\right), \\
\hat{k}(s) & =B e^{-c s} \prod_{j=0}^{\infty} g_{j}(s) .
\end{aligned}
$$

Lemma 2. If $|t| \geqq j$ then $\left|g_{k}(i t)\right| \geqq 1, k=0,1, \cdots, j$. If $|t| \leqq j$ then $\left|g_{k}(i t)\right| \leqq 1, k=j+1, j+2, \cdots$.

Proof. If $|t| \geqq j$ then for all $k \leqq j$ we have $|t| \geqq j \geqq k$ so that $|t| \geqq S(z)$ for $t$ in $A_{k}$. By Lemma 1 each factor in the product composing $g_{k}$ is at least 1 in absolute value.

For the second part suppose $|t| \leqq j$ and $k \geqq j+1$. Then $|t| \leqq j \leqq k-1$ so that $|t| \leqq S(z)$ for $z \in A_{k}$. Now apply Lemma 1 again.

Using (10) and Lemma 2 it is not difficult to see that

$$
\left|\frac{\hat{k}(i t)}{\prod_{0}^{k} g_{j}(i t)}\right| \leqq \max [|\hat{k}(i t)|,|B|] \text {. }
$$

In fact by Lemma 2 the left side for $t$ fixed will (as a function of $k$ ) first decrease then increase. It is consequently less than or equal to the larger of the values for $k=0$ and $k=\infty$.

4. The main lemma. With each zero $z$ of $k$ we associate a function $h_{z}(x)$ on $0 \leqq x<\infty$ as follows

$$
h_{z}(x)=\left\{\begin{array}{lc}
|z|^{2} \int_{0}^{x} e^{z(x-t)} e^{\bar{z} t} d t, & \text { z imaginary }, \\
-z e^{z x}, & z \text { real. }
\end{array}\right.
$$

These have the respective Laplace transforms

$$
\frac{1}{1-s / z} \frac{1}{1-s / \bar{z}}
$$

and

$$
\frac{1}{1-s / z}
$$

valid for $R s>R z$.

We use $l^{*} m$ to denote the convolution

$$
\int_{0}^{x} l(t) m(x-t) d t=\int_{0}^{x} m(t) l(x-t) d t
$$

of two functions $l$ and $m$, and $\prod^{*}$ to denote the convolution product of several 
functions. Wherever we use such a product it will be obviously associative.

Now define by induction) $\left(^{3}\right)$

$$
\begin{aligned}
& H_{0}(x)=\lim _{m \rightarrow \infty} f(x)^{*} \prod_{i=1 ; z_{i} \in A_{0}}^{m} h_{z_{i}}(x), \\
& H_{k}(x)=\lim _{m \rightarrow \infty} H_{k-1}(x)^{*} \prod_{i=1 ; z_{i} \in A_{k}}^{m} h_{z_{i}}(x),
\end{aligned}
$$

whenever the defining expressions have meaning. Our object in this section is to prove the

MaIn Lemma. The functions $H_{k}(x)$ exist for $0 \leqq x \leqq 2 b$ and

$$
\lim _{\boldsymbol{k} \rightarrow \infty} H_{k}(x)=B \phi(x-c), \quad c \leqq x \leqq 2 b,
$$

where $c$ and $B$ are defined by (8).

We begin by considering the new integral equation

$$
f_{1}(x)=\int_{0}^{x} \phi_{1}(x-t) d k_{1}(t), \quad 0 \leqq x<\infty,
$$

where

and

$$
\phi_{1}(x)=\left\{\begin{array}{cl}
\phi(x), & 0 \leqq x \leqq 2 b \\
\epsilon C^{2}, & 0 \leqq x \leqq 3 b \\
0 & x \geqq 3 b
\end{array}\right.
$$

By comparison with (3)

$$
k_{1}(x)= \begin{cases}k(x), & 0 \leqq x \leqq 2 b, \\ k(2 b), & x \geqq 2 b .\end{cases}
$$

Taking Laplace transforms on both sides of the new integral equation we have

$$
f_{1}(x)=\left\{\begin{array}{cl}
f(x), & 0 \leqq x \leqq 2 b, \\
0, & x \geqq 5 b .
\end{array}\right.
$$

where $\hat{k}(s)$ is defined by (5) and $\hat{f}_{1}, \hat{\phi}_{1}$ by

$$
\int_{0}^{5 b} e^{-8 u} f_{1}(u) d u \text { and } \int_{0}^{2 b} e^{-8 u} \phi_{1}(u) d u
$$

(3) In (12) and similar products we take $\Pi^{*}$ to have no effect on the other factor if the corresponding $A_{j}$ is empty. 
respectively. Laplace transforms in general will be denoted by the roof symbol -

In view of assumptions $\mathrm{B} 1$ and $\mathrm{B} 2$ we know that

$$
\widehat{\phi}_{1}(i t)=O\left(1 / t^{2}\right) \text {, }
$$$$
|t| \rightarrow \infty \text {. }
$$

Since $\hat{k}(i t)$ is bounded (15) yields

$$
\hat{f}_{1}(i t)=O\left(1 / t^{2}\right), \quad|t| \rightarrow \infty .
$$

The Main Lemma will be a consequence of the next one. First define the functions $L_{k}(x)$ just as the functions $H_{k}(x)$ are defined in (12), but replacing $f$ by $f_{1}$. Then by (14)

$$
L_{k}(x)=H_{k}(x), \quad 0 \leqq x \leqq 2 b .
$$

Secondly define

$$
\begin{aligned}
& l_{0}(x)=\lim _{m \rightarrow \infty} \int_{0}^{x}\left(\prod_{i=1 ; z_{i} \in A_{0}}^{m} h_{z_{i}}(x-t)\right) d k_{1}(t), \\
& l_{k}(x)=\lim _{m \rightarrow \infty} l_{k-1}(x)^{*} \prod_{i=1 ; z_{i} \in A_{k}}^{m} h_{z_{i}}(x) .
\end{aligned}
$$

Lemma 3. The limits defining $l_{k}(x)$ exist for $k=0,1, \cdots$ boundedly in $0 \leqq x<\infty$; the $l_{k}(x)$ vanish for $x \geqq 2 b$ and their Laplace transforms are

$$
\hat{l}_{k}(s)=\hat{k}(s) / \prod_{j=0}^{k} g_{j}(s),
$$

where $g_{j}$ is defined as in (10). Moreover the functions $L_{k}(x)$ exist for $k=0,1, \cdots$, $0 \leqq x<\infty$ and equal the absolutely convergent integrals

$$
L_{k}(x)=\frac{1}{2 \pi} \int_{-\infty}^{\infty} e^{i t x} \widehat{\phi}_{1}(i t) \hat{l}_{k}(i t) d t .
$$

This will be proved in the next section. Before doing so we show how it is used in proving the Main Lemma. By combining (18), (19) and (20) we have

$$
H_{k}(x)=\frac{1}{2 \pi} \int_{-\infty}^{\infty} e^{i t x} \widehat{\phi}_{1}(i t) \frac{\hat{k}(i t)}{\prod_{j=0}^{k} g_{j}(i t)} d t, \quad 0 \leqq x \leqq 2 b .
$$

By virtue of (11) and (16) we may let $k \rightarrow \infty$ under the integral. (10) tells us then that for $x>c$

$$
\lim _{k \rightarrow \infty} H_{k}(x)=\frac{1}{2 \pi} \int_{-\infty}^{\infty} e^{i t x} \widehat{\phi}_{1}(i t) B e^{-c i t} d t=B \phi_{1}(x-c) .
$$


Since $\phi_{1}$ and $\phi$ agree on $0 \leqq x \leqq 2 b$, this establishes (13).

5. Proof of Lemma 3. The assertion about $l_{k}(x)$ will be proved by induction on $k$. We start with $k=0$. Define for $m \geqq 1$

$$
l_{0}^{(m)}(x)=\int_{0}^{x}\left(\prod_{i=1 ; z_{i} \in A_{0}}^{m} h_{z_{i}}(x-t)\right) d k_{1}(t) .
$$

Taking Laplace transforms we obtain

$$
l_{0}^{(m)}(s)=\hat{k}_{1}(s)\left\{\prod_{i=1 ; z_{i} \in A_{0}}^{m}\left(1-\frac{s}{z_{i}}\right)\left(1-\frac{s}{\bar{z}_{i}}\right)\right\}^{-1} .
$$

By the complex inversion formula for Laplace transforms

$$
l_{0}^{(m)}(x)=\frac{1}{2 \pi i} \int_{d-i \infty}^{d+i \infty} e^{s x} \hat{k}(s)\{\cdots\}^{-1} d s
$$

if $d>R z_{1}, R z_{2}, \cdots, R z_{m}$. By Cauchy's theorem we may move the line of integration to $d=0$ since the integrand is entire and sufficiently small at $\pm i \infty$. Hence

$$
l_{0}^{(m)}(x)=\frac{1}{2 \pi} \int_{-\infty}^{\infty} e^{i t x} \hat{k}(i l)\left\{\prod_{j=1}^{m}\left(1-\frac{i l}{z_{j}}\right)\left(1-\frac{i l}{\bar{z}_{j}}\right)\right\}^{-1} d l .
$$

By Lemma $1 \lim _{m \rightarrow \infty} l_{0}^{(m)}(x)$ exists boundedly as $m \rightarrow \infty$.

To prove that $l_{0}(x)$ vanishes outside $(0,2 b)$ it is enough to prove it for each $l_{0}^{(m)}(x)$. We prove it by induction noting that

$$
\begin{aligned}
& l_{0}^{(1)}(x)=\int_{0}^{x} h_{z_{1}}(x-t) d k_{1}(t), \\
& l_{0}^{(m)}(x)=l_{0}^{(m-1)}(x)^{*} h_{z_{m}}(x) .
\end{aligned}
$$

Consider first $l_{0}^{(1)}(x)$. If $z_{1}$ is real we have

$$
\begin{aligned}
l_{0}^{(1)}(x) & =z_{1} \int_{0}^{x} e^{(x-t) z_{1}} d k_{1}(t), \\
& =z_{1} e^{x z_{1}} \int_{0}^{x} e^{-t z_{1}} d k_{1}(t) .
\end{aligned}
$$

Now if $x \geqq 2 b$ the last integral is

$$
\int_{0}^{2 b} e^{-t z 1} d k(t)
$$

since $k_{1}(x)$ and $k(x)$ agree on $(0,2 b)$. But this vanishes since it is $k\left(z_{1}\right)$ and $z_{1}$ is a zero of $k(s)$. If $z_{1}$ is not real we note that 


$$
h_{z_{1}}(x)=\left(-z_{1} e^{z_{1} x}\right)^{*}\left(-\bar{z}_{1} e^{\bar{z}_{1} x}\right)
$$

so that a similar argument applies. Remember that $\bar{z}_{i}$ is also a zero of $\hat{k}(s)$.

Now suppose, to continue this induction that $l_{0}^{(m-1)}(x)$ vanishes for $x \geqq 2 b$. We have

$$
l_{0}^{(m)}(x)=\int_{0}^{x} h_{z_{m}}(x-t) l_{0}^{(m-1)}(t) d t
$$

If $x \geqq 2 b$

$$
l_{0}^{(m)}(x)=\int_{0}^{2 b} h_{z_{m}}(x-t) l_{0}^{(m-1)}(t) d t .
$$

The argument used for $m=1$ still holds provided $z_{m}$ and $\bar{z}_{m}$ are zeros of $\ell_{0}^{(m-1)}(s)$. Since they are zeros of $k(s)$, this follows from (21) with $m$ replaced by $m-1$.

To complete the case $k=0$ we must establish (19), i.e. that

$$
\eta_{0}(s)=\hat{k}(s) / g_{0}(s) \text {. }
$$

We know that

$$
l_{0}^{(m)}(s)=\int_{0}^{2 b} e^{-8 u} l_{0}^{(m)}(u) d u
$$

since $l_{0}^{(m)}$ vanishes outside $(0,2 b)$. By bounded convergence

$$
\lim _{m \rightarrow \infty} l_{0}^{(m)}(s) \int_{0}^{2 b} e^{-s u} l_{0}(u) d u=l_{0}(s) .
$$

Now compare (21).

To continue the induction on $k$ suppose the assertions of the lemma hold for $k-1$. Then defining

$$
l_{k}^{(m)}(x)=l_{k-1}(x)^{*} \prod_{i=1 ; z_{i} \in A_{k}}^{m} h_{z_{i}}(x)
$$

we know that

$$
\lim _{m \rightarrow \infty} l_{k-1}^{(m)}(x)=l_{k-1}(x) \text { boundedly, }
$$

that

$$
l_{k-1}(s)=\int_{0}^{2 b} e^{-s u} l_{k-1}(u) d u
$$

and that 


$$
l_{k-1}(s)=\frac{\hat{k}(s)}{\prod_{j=0}^{k-1} g_{i}(s)}
$$

According to (22) we have

$$
l_{k}^{(m)}(s)=l_{k-1}(s)\left\{\prod_{i=1 ; z_{i} \in A_{k}}^{m}\left(1-\frac{s}{z_{i}}\right)\left(1-\frac{s}{\bar{z}_{i}}\right)\right\}^{-1} .
$$

We now complete the proof as for $k=0$ with the following changes. At the stage where Cauchy's theorem is used to move the line of investigation use the boundedness of $\hat{l}_{k-1}(s)$ in $0 \leqq R s \leqq d$. This follows from (23). To prove the bounded existence of $\lim _{m \rightarrow \infty} l_{k}^{(m)}(x)$ use Lemma 2 in place of Lemma 1. To prove the vanishing of $l_{\boldsymbol{k}}^{(m)}(x)$ outside of $(0,2 b)$ we employ an induction as before, noting that by (24) the elements of $A_{k}$ are zeros of $\hat{l}_{k-1}(s)$. The details are left to the reader.

It remains to prove the assertion of Lemma 3 concerning $L_{k}(x)$. By (13a) and the associative property of convolution it follows from the definition of $L_{k}(x)$ (see the sentence preceding (18)) that

$$
L_{0}(x)=\lim _{m \rightarrow \infty} \phi_{1} * l_{0}^{(m)}(x),
$$

so that by Lemma 3

$$
L_{0}(x)=\phi_{1} * l_{0}(x)
$$

By induction

$$
L_{k}(x)=\phi_{1} * l_{k}(x)
$$

for suppose

$$
L_{k-1}(x)=\phi_{1} * l_{k-1}(x)
$$

Then

$$
\begin{aligned}
L_{k}(x) & =\lim _{m \rightarrow \infty} L_{k-1}(x) * \prod_{i=1 ; z_{i} \in A_{k}}^{m} h_{z_{i}}(x) \\
& =\lim _{m \rightarrow \infty} \phi_{1}(x) * l_{k-1}(x) * \prod_{i=1 ; z_{i} \in A_{k}}^{m} h_{z_{i}}(x) \\
& =\lim _{m \rightarrow \infty} \phi_{1}(z) * l_{k}^{(m)}(x) \\
& =\phi_{1}(x) * l_{k}(x)
\end{aligned}
$$

by Lemma 3 again. This completes the inductive proof of (25). By (25) 


$$
\begin{aligned}
\hat{L}_{k}(s) & =\widehat{\phi}_{1}(s) \hat{l}_{k}(s), \\
L_{k}(x) & =\frac{1}{2 \pi i} \int_{d-i \infty}^{d+i \infty} e^{s x} \widehat{\phi}_{1}(s) \eta_{k}(s) d s
\end{aligned}
$$

for $d>0$ and now (20) follows since the line of integration can be moved once again to $d=0$.

6. Recapitulation. In view of the mass of details let us restate where matters stand. We begin with (3) as given.

Theorem 1. Let $b$ be a number such that $0<b<a / 2$ and such that (4) holds. Define $\hat{k}$ by (5) and $c$ as in (8). Arrange the roots of $\hat{k}$ into the classes $A_{n}$ described in §3. Define $h_{z}(x)$ as in $\$ 4$ and $H_{k}(x)$ as in (12). Then (3) has the solution

$$
\lim _{k \rightarrow \infty} \frac{1}{B} H_{k}(x+c)=\phi(x), \quad 0 \leqq x \leqq b .
$$

(26) is a restatement of (13) and (9). The solution is valid in the (possibly) larger interval $0 \leqq x \leqq 2 b-c$, but this fact will not be useful for our ultimate goal which is a solution valid in $0 \leqq x<a$. This is described in the following section.

Note that (26) uses the values of $f(x)$ in at most the interval $0 \leqq x \leqq 2 b$. This is due to the definition of $H_{0}(x)$ in (12).

7. The solution completed. It is now our object to show how the integral equation in (3) can be solved in any interval $0 \leqq x \leqq a-\eta, 0<\eta<a$, by a finite number of repetitions of the procedure described in Theorem 1 . Choose $b$ so small that (4) holds, and that two of the successive numbers $b, 2 b, 3 b, \cdots$ fall into the interval $a-\eta \leqq x<a$, say

$$
a-\eta \leqq N b \mathbf{i}<(N+1) b<a .
$$

If $N=1$ Theorem 1 accomplishes the solution so suppose $N>1$.

We shall show how to use the procedure of Theorem 1 to find $\phi(x)$ in an interval $0 \leqq x \leqq(n+1) b$, given the solution in $0 \leqq x \leqq n b, 1 \leqq n \leqq N-1$. Since $\phi$ is given in $0 \leqq x \leqq b$ by Theorem 1 , an $N$-fold repetition of the procedure gives the solution in $0 \leqq x \leqq N b$ and this includes the prescribed interval $0 \leqq x \leqq a-\eta$.

Define

$$
f_{n}(x)=f(x)+\int_{0}^{n b} \phi(t) d k(x-t)
$$

(3) takes the form

$$
f_{n}(x)=-\int_{n b}^{x} \phi(t) d k(x-t)
$$


valid if $n b \leqq x<a$. Replace $x$ by $x+n b$. Then

$$
f_{n}(x+n b)=-\int_{n b}^{x+n b} \phi(t) d k(x+n b-t),
$$

valid if $0 \leqq x<a-n b$. Changing variables we find that

$$
f_{n}(x+n b)=\int_{0}^{x} \phi(s+n b-t) d k(t)
$$

valid in $0 \leqq x<a-n b$, hence, by (27) in $0 \leqq x \leqq 2 b$. This final integral equation is of the same type as the original, so that the method of Theorem 1 applied with $f_{n}(x+n b)$ in place of $f(x)$ yields $\phi(x+n b)$ in $0 \leqq x \leqq b$, and hence $\phi(x)$ in $n b \leqq x \leqq(n+1) b$. Since $\phi(x)$ is known in $0 \leqq x \leqq n b$, this completes the program.

\section{REFERENCES}

1. E. C. Titchmarsh, Introduction to the theory of Fourier integrals, 1st ed., Oxford, 1937.

2. - The theory of functions, Oxford, 1939.

Cornell University,

ITHACA, N.Y. 